\title{
Atlanto-axial Joint Distraction in the Treatment of Select Cases of Basilar Invagination, Syringomyelia and Fixed Atlanto-axial Dislocation
}

Atul Goel, MCh

Professor and Head

Department of Neurosurgery

K.E.M. Hospital and Seth G.S. Medical College

Parel, Mumbai

India

Email: atu169@hotmail.com

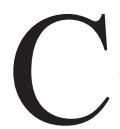
raniovertebral anomalies present an array of complex bony malformations leading to a range of symptoms secondary to neural compression, malalignment of bone and subtle or manifest instability. The treatment of these malformations is a challenging problem. Whilst reduction and fixation in the reduced position of mobile and reducible atlanto-axial dislocation is the standard and accepted form of treatment, the treatment of anomalies like basilar invagination, syringomyelia and 'fixed' atlantoaxial dislocation remains under discussion. Restoration of the distorted alignments without any bony decompression, dural or neural manipulation is possible and probably an ideal form of treatment in a select group of these anomalies.

Congenital craniovertebral anomalies are significantly more common in the Indian subcontinent than in other parts of the world. The exact reason for this disproportionately high incidence is not clear. No definite genetic anomaly has been identified. Even in India, the general observation is that the relatively poor population is more frequently affected.

The bone development is a late embryogenic event. The entire basic neural and vascular tree of the fetus is formed much before the formation of even a spicule of bone. The formation of the craniovertebral junction and its alignment is in the process of completion during the birth of the fetus and also after birth. The gross alignment is completed after the infant starts holding the head at the age of about 3-4 months. 'Basilar invagination' is normally present in the fetus. It is after the reversal of the cervical flexure, formation of the craniovertebral and spheno-occipital sclerotomes, occipital condyle and suboccipital bone, facets of the atlas and axis that the odontoid process descends down in relationship to the neural structures and 'basilar invagination' is reversed. Basilar invagination could be a result of errors in the embryological development where the formation of craniovertebral bones necessary to reverse the fetal basilar invagination is incomplete or inadequate. In most of the congenital craniovertebral anomalies the neural and vascular formation is normal and complete. The role of fetal malnutrition, inadequate strength of the muscles of the neck or improper fetal delivery practices in the development of craniovertebral anomalies is not clear. However, these factors could have a bearing on the pathogenesis of basilar invagination, as the probable time of initiation of the dysgenesis is during and around the period of delivery of the fetus.

Basilar invagination could be a price that humans are paying for their erect standing position. The distribution of the weight of the head and alteration of its center of gravity due to bony malformation could result in a progressive strain on the muscles of the nape of the neck, instability and subsequent mal-alignment.

On the basis of the literature review and our own study, ${ }^{8}$ we have observed that the maldevelopment results in a reduced length of the clivus (i.e. the sphenoid part of the clivus is formed relatively normally, whereas the occipital part is formed incompletely) and platybasia, occipital condylar hypoplasia, non-formation or inadequate formation of the occipito-axial joint, and frequently, occipitalization of the atlas. Fusion of the atlanto-axial joint, and C2-3 spinal elements and a range of Klippel-Feil spinal abnormalities are also frequently associated. The entire complex of the odontoid process, the atlas, and the clivus is rostrally located, and effectively the volume of the posterior cranial of fossa is reduced. Partial or complete assimilation of the atlas is an important and frequent component of the mesodermal maldevelopment. Basal mesodermal maldevelopment will result in rostral positioning of the plane of the foramen magnum and significantly severe basilar invagination if measurements are taken on the basis of parameter laid down by Chamberlain. ${ }^{2}$ However, the tip of the odontoid process will remain below both Wackenheim's clival line and McRae's line of the foramen magnum in a large percentage of cases. ${ }^{18,19}$

Chiari malformation ${ }^{3}$ and related pathological events could be primarily attributable to maldevelopment of the occipital bone and overcrowding of the normally developed cerebellum within a smaller posterior cranial fossa. ${ }^{8}$ There is usually no demonstrable structural abnormality of the brainstem, cerebellar hemisphere or fourth ventricle suggesting that the neural development in these patients was unaffected in the embryonic dysgenesis. Chiari malformation or the tonsillar herniation appears to be the result of the presence of normal cerebellar mass in a smaller posterior cranial fossa. Long-standing pulsatile pressure of the herniating tonsil to the brainstem as a cause of formation of syringomyelia cavitation has been discussed. Essentially, it appears that syringomyelia is a tertiary event to the primary basilar invagination and secondary Chiari malformation. Our observations suggest that the treatment strategy in such cases should be directed towards increasing the posterior 


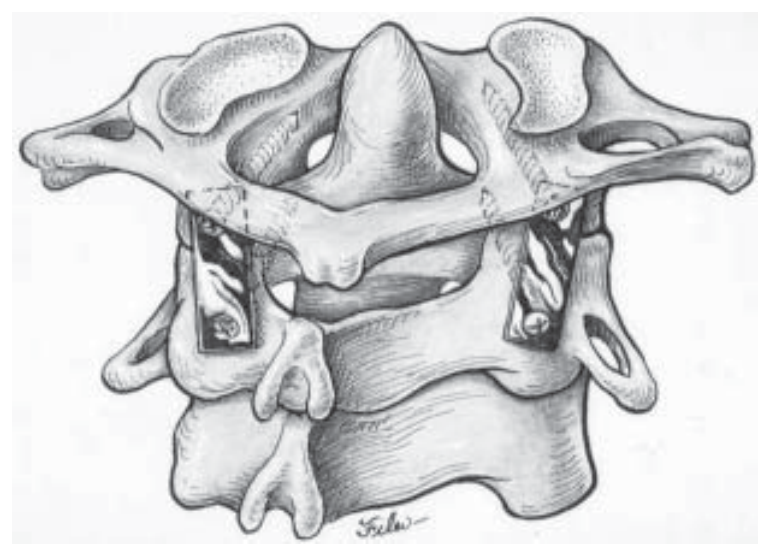

Figure 1. Line diagram of the construct showing the plate and screw technique of inter-articular atlanto-axial fixation.

cranial fossa volume by foramen magnum decompression, which is the primary pathology. Opening of the posterior cranial fossa dura, resection of the cerebellar tonsils, arachnoidal sectioning and syringostomy can be avoided. It appears that if the primary problem is dealt with, the secondary and the tertiary events will spontaneously regress. ${ }^{8}$ The theory that dura or dural bands can act as compressive factor appears unacceptable and does not correlate with the pathogenic events. Dura is an expansile structure and is unlikely to be a compressive factor. Our observation is that if the syringomyelia is treated without dealing with the primary pathology, more often than not the outcome will be poor. ${ }^{5,9}$

\section{Treatment of Selected Cases of Congenital Basilar Invagination}

We had discussed the subject of basilar invagination and had presented a classification for these anomalies based on the presence or absence of Chiari I malformation. ${ }^{8}$ On the basis of the possible pathogenetic factors, we had suggested a specific treatment protocol for each of the two groups. With our improved understanding of the subject, we reclassified basilar invagination into two groups, Group A and Group B, based on parameters that determined an alternative treatment strategy. ${ }^{6}$ In Group A basilar invagination there was a 'fixed' atlanto-axial dislocation and the tip of the odontoid process 'invaginated' into the foramen magnum and was above the Chamberlain line, ${ }^{2}$ McRae line of foramen magnum, ${ }^{18}$ and Wackenheim's clival line. ${ }^{19}$ The definition of basilar invagination of prolapse of the cervical spine into the base of the skull, as suggested by von Torklus, ${ }^{20}$ was suitable for this group of patients. We reviewed our entire series of cases with basilar invagination and identified that Group A basilar invagination constitutes approximately $60 \%$ of all cases of basilar invagination.

The majority of patients with Group A basilar invagination had a history of minor to major head injury prior to the onset of the symptoms. The pyramidal symptoms and affection of the kinesthetic sensations formed a dominant component. Spinothalamic dysfunction was less frequent. Neck pain as a major presenting symptom was noted in approximately $75 \%$ cases. Torticollis was present in approximately $50 \%$ cases. The analysis of clinical features suggests that the symptoms and signs in this group of patients were a result of brainstem compression by the odontoid process. The radiological features suggested that the odontoid process resulted in direct compression of the brainstem. Analysis on the basis of Chamberlain's line showed that the basilar invagination was mild to severe in these cases. Modified omega angle measurements suggested that the odontoid process had tilted towards the horizontal plane rather than rostrally. ${ }^{17}$ The patients had a 'fixed' atlanto-axial dislocation and no atlanto-axial mobility could be identified on dynamic radiology in any case.

The standard and most accepted form of treatment of Group A basilar invagination is a transoral decompression. The majority of authors recommend a posterior occipitocervical fixation following the anterior decompression. It appears to us that the atlanto-axial joint in such cases is in an abnormal position as a result of congenital abnormality of the bones and progressive worsening of the dislocation is probably secondary to increasing 'slippage' of the atlas over the axis. The slip of the atlas over the axis appears to be accentuated by the event of trauma. With our experience in handling the atlantoaxial joints, we have realized that the joint in these cases is not 'fixed' or 'fused' but is mobile and in some cases is hypermobile, and is probably the prime cause for the basilar invagination. The history of trauma preceding the clinical events, predominant complaint of pain in the neck and the improvement in neurological symptoms following institution of cervical traction suggests 'vertical' instability of the craniovertebral region.

We had earlier attempted to reduce basilar invagination by performing occipitocervical fixation following institution of cervical traction. ${ }^{8}$ However, all the 4 cases treated in this
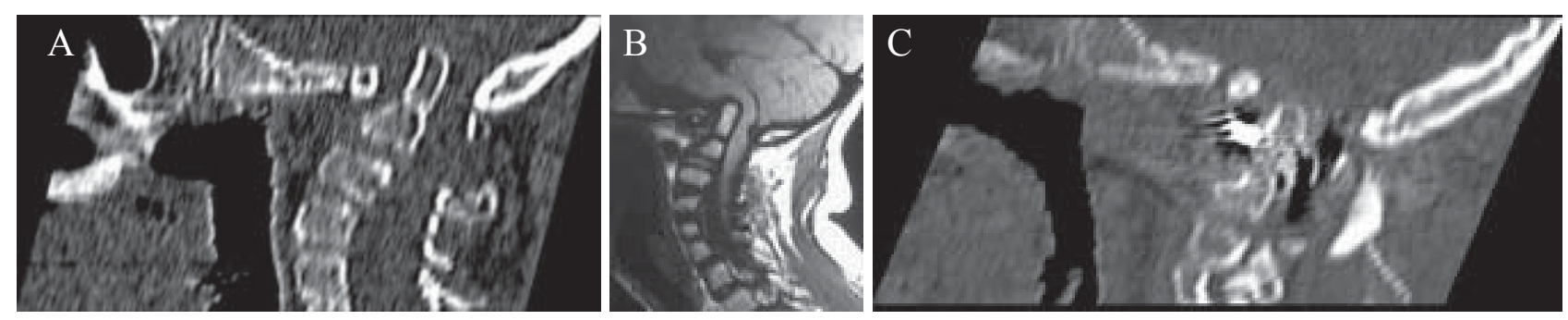

Figure 2. Treatment of basilar invagination. A) CT scan of craniovertebral junction of a 12-year old boy showing marked basilar invagination. B) MRI showing severe indentation of the cervicomedullary cord by the odontoid process. C) Postoperative CT scan showing distraction of the atlanto-axial joint and craniovertebral realignment 

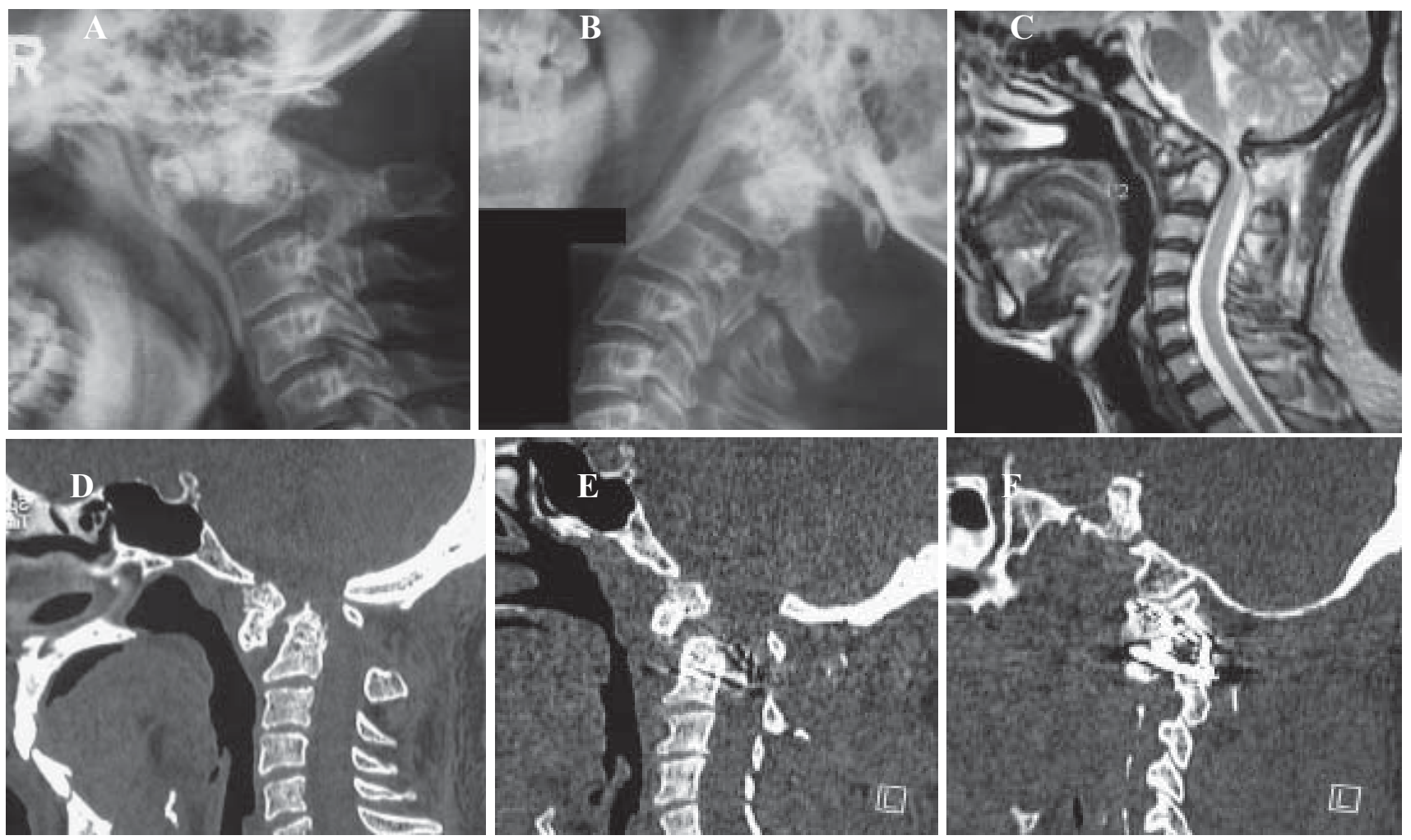

Figure 3. Treatment of fixed atlanto-axial dislocation. A) Lateral X-ray of the craniovertebral region with flexion of the neck in a 22-year-old man showing atlanto-axial dislocation. B) Lateral X-ray in extension shows no reduction of the dislocation. C) T2-weighted MRI showing severe cord compression. D) CT scan showing the atlanto-axial dislocation. Osodontoideum can be seen. E) Post-operative CT scan showing reduction of the dislocation. F) Post-operative CT scan with a cut through the atlanto-axial joint. The plate and screws and the spacer can be seen.

manner subsequently needed transoral decompression as the reduction of the basilar invagination and of atlanto-axial dislocation could not be sustained by the implant. Wide removal of atlanto-axial joint capsule and articular cartilage by drilling and subsequent distraction of the joint by manual manipulation provided a unique opportunity to obtain reduction of the basilar invagination and of atlanto-axial dislocation. The joints were maintained in a distracted and reduced position with the help of bone graft and spacers. The subsequent fixation of the joint with the help of interarticular screws and a metal plate (Figure 1) provided a biomechanically firm fixation. ${ }^{10,12,14}$ The fixation was seen to be strong enough to sustain the vertical, transverse and rotatory strains of the most mobile region of the spine. Following surgery, the alignment of the odontoid process and the clivus and the entire craniovertebral junction improved towards normalcy (Figure 2). The tip of the odontoid process receded in relationship to the Wackenheim's clival line, Chamberlain's line and Macrae's line suggesting reduction in the basilar invagination. The posterior tilt of the odontoid process, as evaluated by modified omega angle, was reduced after the surgery. We could obtain varying degrees of reduction of the basilar invagination and atlanto-axial dislocation. The extent of distraction of the joint and the subsequent reduction in the basilar invagination was more significant in younger than in older patients.

Posterior fossa bony decompression appears to be an ideal form of treatment for Group B basilar invagination.

\section{Treatment of Fixed Atlanto-axial Dislocation (Without Basilar Invagination)}

Atlanto-axial dislocation has been described as 'fixed' or 'irreducible' when there is no radiographic reduction of the dislocation on full neck extension or after institution of cervical traction. Fixed atlanto-axial dislocation can be congenital in nature or can be secondary to trauma to the region. Congenital os odontoideum and fracture at the base of the odontoid process are frequent accompaniments of fixed atlanto-axial dislocation. Various authors have suggested a transoral decompression followed by a posterior fixation as the safest method of treatment of this complex anomaly. Treatment by posterior decompressive procedures has been reported to be associated with a high complication rate. Some authors have reported success with a transoral decompression of the region, without any posterior fixation.

We observed that direct facet joint distraction results in reduction of the fixed dislocation in a significant number of cases. Introduction of bone graft and hydroxyapatite or multi-hole titanium blocks filled with cancellous bone into the joint and fixation with plate and direct implantation of screws in the atlas and axis has the strength to sustain the reduction and provide a ground for a solid bone fusion. With our experience, we feel that there may be a place for reduction of the 'fixed' atlantoaxial dislocation and a subsequent fixation, without the removal of any bony spinal element. ${ }^{13}$ 

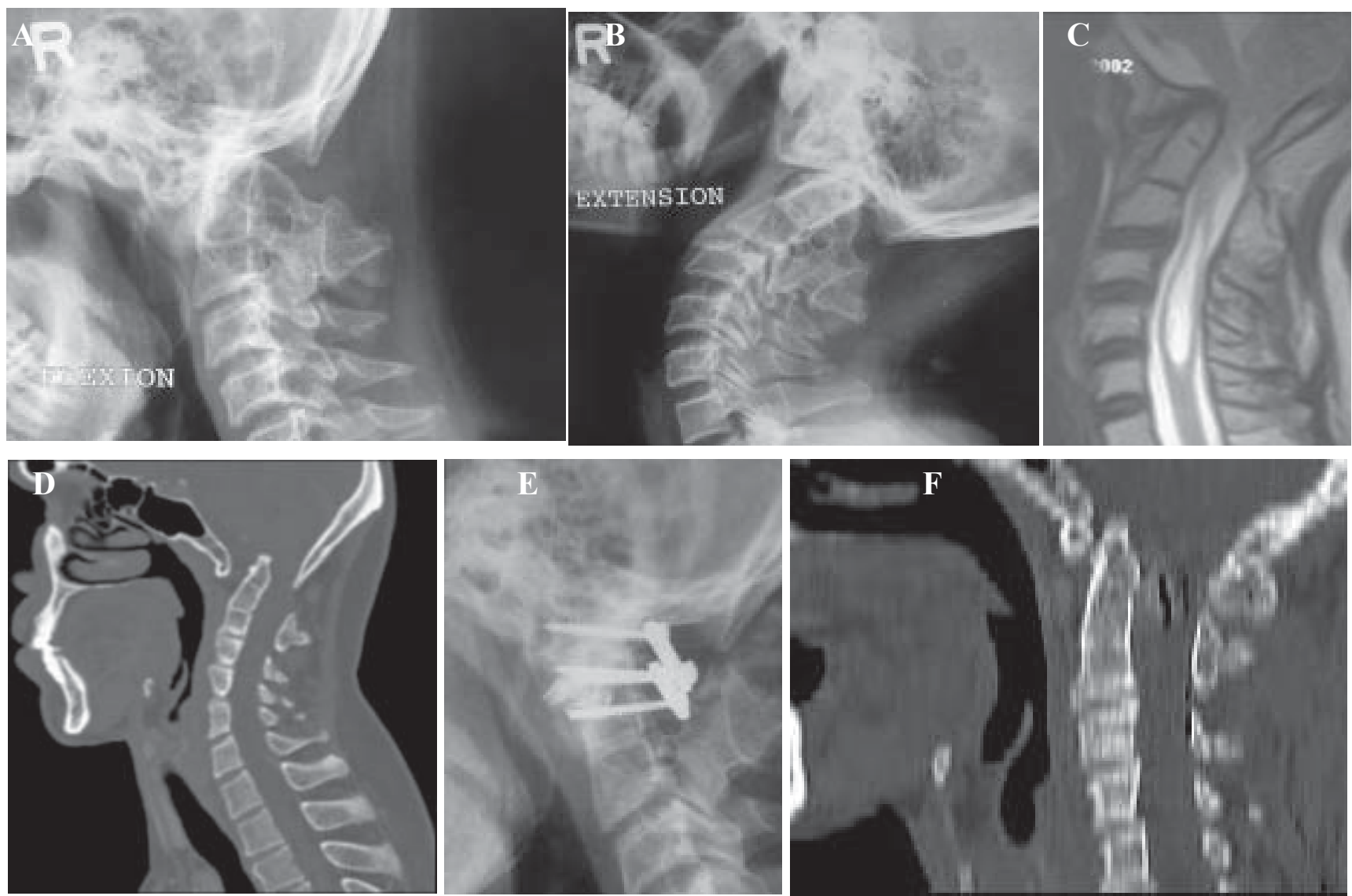

Figure 4. Treatment of syringomyelia. A) Preoperative X-ray of the craniovertebral region of a 48-year-old man with the neck in a flexed position shows occipitalization of the atlas, fusion of the C2-3 vertebra, atlanto-axial dislocation and basilar invagination. B) Preoperative X-ray of the craniovertebral region with the neck in extension position showing persistent atlanto-axial dislocation. C) Preoperative T2-weighted MRI showing basilar invagination, fixed atlanto-axial dislocation, Chiari 1 malformation and syringomyelia. D) CT scan showing the basilar invagination, fixed atlanto-axial dislocation, occipitalization of atlas and fusion of C2-3 vertebra. E) Post-operative X-ray with the neck in flexion showing the fixation with plates and screws. Spacers can be identified within the joint cavity. F) Postoperative CT scan showing the reduction of the basilar invagination and of the atlanto-axial dislocation.

The procedure is technically demanding and anatomically precise, ${ }^{1,4,16}$ but if it is learned adequately and performed successfully, the neurological outcome is extremely gratifying (Figure 3).

\section{Treatment of Selected Cases of Syringomyelia}

We had classified syringomyelia in three groups and had suggested a specific treatment protocol on the basis of the possible pathogenetic factors. ${ }^{5,9}$ The complex of basilar invagination, Chiari 1 malformation and syringomyelia is relatively common and there are multiple reports on the subject. Association of fixed atlanto-axial dislocation in the later group is less common but not rare. Such cases are generally treated by either anterior transoral or posterior foramen magnum bony decompression. The indications and need for opening of the dura and manipulation of arachnoid membrane, tonsils and obex and draining of the syrinx cavity are currently under debate. There are no reports in the literature identifying the need for a specific fixation procedure for cases of fixed atlanto-axial dislocation where it is associated with basilar invagination in the presence of syringomyelia.
It was observed that cases of syringomyelia where there was 'fixed' atlanto-axial dislocation with or without the association of basilar invagination and Chiari malformation constituted a discrete pathologic group. It was observed that in this group, the patients were relatively young, neck pain formed a part of the symptom complex, and the motor symptoms and ataxia was a far more prominent symptom. We observed that an attempt could be made to realign the bones in the craniovertebral junction in these cases, without resorting to any bony or dural decompression or neural manipulation of any kind (Figure 4). The fact that there was a remarkable clinical improvement following the reduction of the atlanto-axial dislocation and of basilar invagination, it appears that the complex of atlanto-axial dislocation, basilar invagination and syringomyelia are probably secondary to the primary craniovertebral instability.

\section{Treatment of Torticollis}

Torticollis is a common symptom in all forms of basilar invagination. No specific treatment modality has been described to treat this symptom. Transoral decompression 

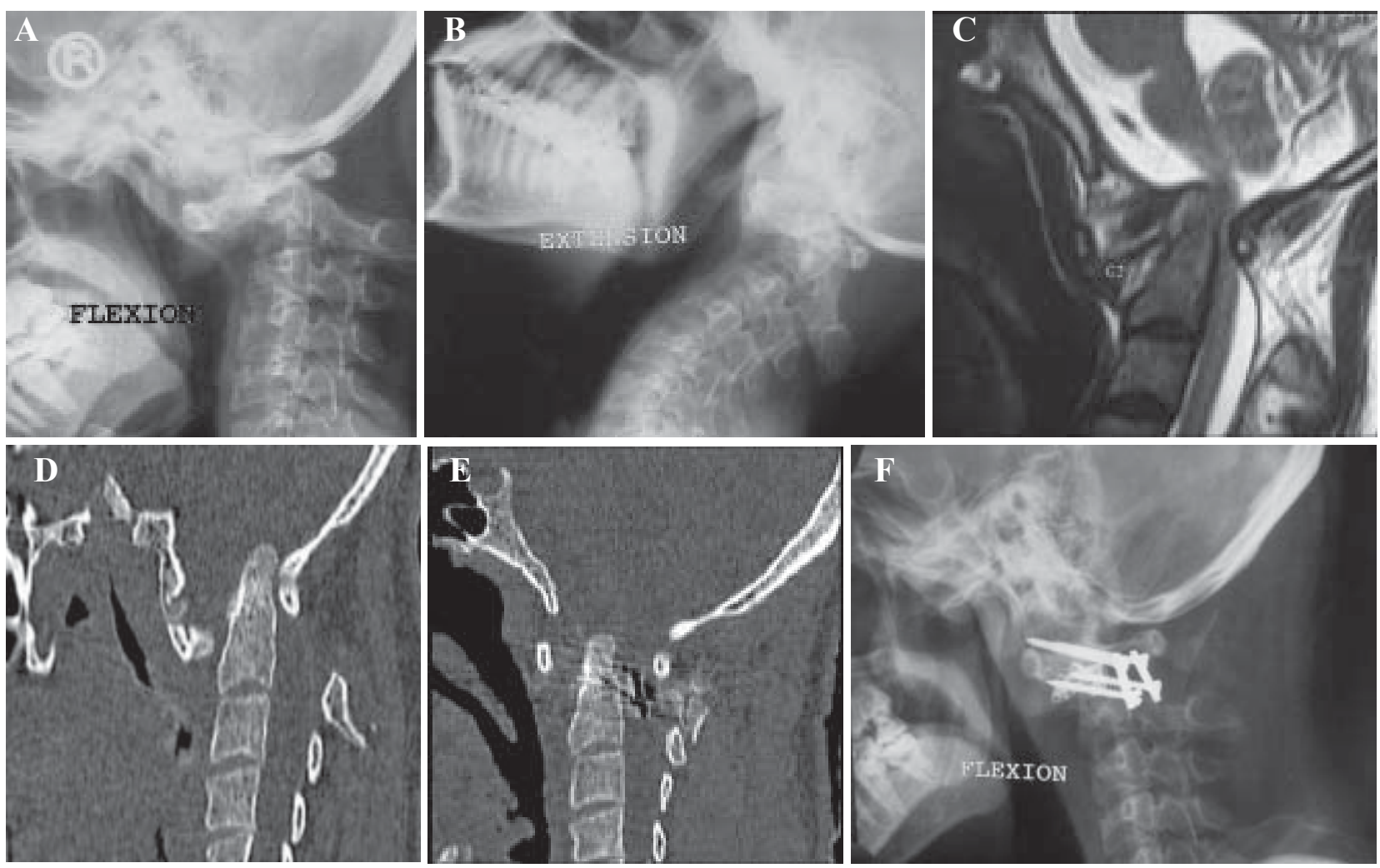

Figure 5. Treatment of basilar invagination and fixed atlanto-axial dislocation in a patient with rheumatoid arthritis. A) Plain X-ray of a 24 year old man, with the head in flexion showing marked atlanto-axial dislocation. B) X-ray with the head in extension showing persistent atlanto-axial dislocation. C) T2-weighted MRI showing basilar invagination and atlanto-axial dislocation and marked compression of the cervicomedullary cord. D) CT scan showing severe atlantoaxial dislocation and basilar invagination. E) Postoperative CT scan showing partial but significant reduction of both atlanto-axial dislocation and of basilar invagination. F) Post-operative X-ray showing atlanto-axial fixation with plate and screws and spacers.

or a posterior fixation procedure can provide decompression of the region and fixation, but the torticollis remains untreated. ${ }^{11}$ We observed that reduction of the atlanto-axial dislocation and of basilar invagination by manual distraction of the atlanto-axial joint presents an ideal opportunity for reduction of the torticollis. Although differential distraction of the joints was not attempted in our patients, this procedure may provide an additional reduction of the torticollis.

\section{Treatment of Basilar Invagination and Atlanto-axial Dislocation in Cases with Rheumatoid Arthritis}

Basilar invagination is commonly associated with atlanto-axial dislocation and the complex results in a significant degree of neck pain and myelopathy adding considerably to the disability secondary to affection of other joints. We recently reported the feasibility of craniovertebral region bone alignment, distraction of the facets of atlas and axis and direct lateral mass plate and screw atlanto-axial fixation for management of both basilar invagination and atlanto-axial dislocation secondary to rheumatoid arthritis. ${ }^{15}$ Our operation of craniovertebral realignment and stabilization without any bone decompression could be successfully employed in cases with atlanto-axial dislocation in the presence or absence of retro-odontoid pannus and in cases with basilar invagination. The age of the patients in our series was relatively less when compared to the similar subset reported in the literature. We observed that the bone quality of the atlas and axis facets for purchase of the screws was satisfactory, allowing the possibility of distraction and stabilization with screw implantation (Figure 5). None of the patients had lateral mass collapse, as has been identified by some authors in their similar cases. The patients showed a remarkable and sustained neurological and radiological improvement. However, it was not possible to assess the extent of regression of pannus in our cases as stainless steel metal plates were used for fixation.

\section{References}

1. Cacciola F, Phalke U, Goel A: Vertebral artery in relationship to $\mathrm{C} 1-\mathrm{C} 2$ vertebrae: An anatomical study. Neurol India 52:178-184, 2004

2. Chamberlain WE: Basilar impression (platybasia). A bizarre developmental anomaly of the occipital bone and upper cervical spine with striking and misleading neurologic manifestations. Yale J Biol Med 11:487-496, 1939

3. Chiari H: Ueber Verderbungen des Kleinhirns infolge von Hydrocephalie des Grossihirns. 
Dwochenschr 17:1172-1175, 1891

4. Goel A: Vertebral artery injury with transarticular screws. J Neurosurg 90:376,1999 (Letter)

5. Goel A: Is syringomyelia pathology or a natural protective phenomenon? J Postgrad Med 47:8788,2001

6. Goel A: Treatment of basilar invagination by atlanto-axial joint distraction and direct lateral mass fixation. J Neurosurg Spine 1:281-286, 2004

7. Goel A, Achawal S: Surgical treatment for Arnold Chiari malformation associated with atlanto-axial dislocation. Br J Neurosurg 9:67-72, 1995

8. Goel A, Bhatjiwale M, Desai K: Basilar invagination: a study based on 190 surgically treated cases. J Neurosurg 88:962-968, 1998

9. Goel A, Desai KI: Surgery for syringomyelia: An analysis based on 163 surgical cases. Acta Neurochir (Wien) 142:293-302, 2000

10. Goel A, Desai K, Muzumdar D: Atlanto-axial fixation using plate and screw method: A report of 160 treated patients. Neurosurgery 51:1351-1357, 2002

11. Goel A, Karapurkar AP: Transoral plate and screw fixation of the clivus to the cervical body. $\mathbf{B r} \mathbf{J}$ Neurosurg 8:743-745,1994

12. Goel A, Kulkarni AG: Mobile and reducible atlantoaxial dislocation in presence of occipitalized atlas: Report on treatment of eight cases by direct lateral mass plate and screw fixation. Spine

\section{9:E520-E523, 2004}

13. Goel A, Kulkarni AG, Sharma P: Reduction of fixed atlantoaxial dislocation: A report of an experience with 24 cases. J Neurosurg (Spine) (in press).

14. Goel A, Laheri VK: Plate and screw fixation for atlanto-axial dislocation. (Technical report). Acta Neurochir (Wien) 129:47-53, 1994

15. Goel A, Sharma P: Craniovertebral realignment for basilar invagination and atlanto-axial dislocation secondary to rheumatoid arthritis. Neurol India 52:338-41, 2004

16. Gupta S, Goel A: Quantitative anatomy of lateral masses of the atlas and axis vertebrae. Neurol India 48:120-125, 2000

17. Klaus E: Rontgendiagnostik der platybasic und basilar Impression. Fortschr Rontgenstr 86:460469, 1957

18. McRae DL: Bony abnormalities in the region of foramen magnum: correlation of anatomic and neurologic findings. Acta Radiol 40:335-354,1953

19. Thiebaut F, Wackenheim A, Vrousos C: New median sagittal pneumostratigraphical findings concerning the posterior fossa. J Radiol Electrol 42:1-7,1961 (French)

20. Von Torklus D, Gehle W: The Upper Cervical Spine: Regional Anatomy, Pathology, and Traumatology. A Systematic Radiological Atlas and Textbook. New York: Grune\& Stratton, 1972, pp 1-98 